\title{
Old concepts and new developments in the study of platelet aggregation
}

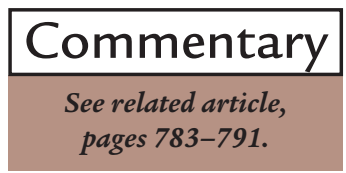

\author{
Zaverio M. Ruggeri
}

The Scripps Research Institute, Molecular and Experimental Medicine-175, 10550 N. Torrey Pines Road, La Jolla, California 92037, USA. Phone: (858) 784-8950; Fax: (858) 784-2026; E-mail: ruggeri@scripps.edu.

Platelets, anucleated cells that originate from the cytoplasm of bone marrow megakaryocytes (1), circulate through the blood, surveying the integrity of the vascular system. Platelets act in response to traumatic injuries in which the continuity of the vessel wall is interrupted and blood begins to pour outside $(2,3)$. The arrest of posttraumatic hemorrhage depends on a complex set of regulated events collectively defined as hemostasis, which include the clotting of fibrinogen into insoluble fibrin, as well as platelet adhesion to exposed tissues and to one another with the formation of masses known as thrombi (4). However, neither platelets nor other components of the hemostatic process can distinguish between traumatic wounds and other lesions that may develop in the vessel wall. Thus, when occlusive thrombi form within the vasculature, an otherwise beneficial function, which limits blood loss after tissue injury, becomes a potentially life-threatening disease mechanism. Such a course of events is usually initiated by destabilizing conditions in arteries affected by chronic degeneration, as when an atherosclerotic plaque suddenly ruptures $(5,6)$. Platelet-rich thrombi that acutely curtail the supply of blood to vital organs may cause death or serious pathological conditions, such as ischemic syndromes of the heart and brain. Knowledge of the mechanisms of platelet function has informed the development of potent and selective antithrombotic drugs, and first-generation antiplatelet compounds that specifically block adhesion receptors have already proved beneficial in the clinic (7-9).

In response to vascular and tissue trauma, platelets establish adhesive interactions with exposed subendothelial structures. They become activated through contact with thrombogenic surfaces or through stimulation by locally released or generated chemical agonists. Once activated, platelets bind soluble adhesive molecules and become the reactive surface for continuing platelet deposition (Figure 1). Initial platelet tethering to a surface and subsequent platelet-platelet cohesion are typically identified as 2 separate stages of thrombus formation, defined as adhesion and aggregation, respectively. In support of this distinction, adhesion requires a more diverse repertoire of substrates and platelet receptors than does aggregation $(10,11)$. For example, thrombus formation initiated by platelet adhesion to extracellular matrix (ECM) involves the synergistic function of at least 4 receptors, the glycoprotein (GP) Ib-IX-V complex and the integrins $\alpha_{2} \beta_{1}$ (GP Ia-IIa), $\alpha_{\text {IIb }} \beta_{3}$ (GP Iib-IIIa), and $\alpha_{5} \beta_{1}$ (GP Ic-IIIa) (10). Aggregation, in contrast, may depend only on the GP Ib-IX-V complex and $\alpha_{\mathrm{IIb}} \beta_{3}(11)$. On the other hand, adhesion and aggregation are similar with respect to the effects of blood flow: To form stable bonds either with ECM components or with other platelets (11), circulating platelets must attach to a reactive substrate, resisting the force of flowing blood, which would tend to move platelets with the layer of fluid adjacent to the vessel wall. During adhesion, subendothelial and extravascular ECM components provide this substrate, and during aggregation activated platelets that are already firmly adherent play this role; but in either case, fluid drag opposes the initial establishment and subsequent enlargement of the thrombus. Consequently, it can be surmised that shear-dependent phenomena are particularly relevant where forces generated by flow are greater, namely in arteries more than in veins and, particularly, in arterioles $(10,11)$.

Experimental models used in the study of platelet aggregation often create conditions that deviate from those in an intact organism. In particular, models of platelet function usually highlight only one or a few aspects of a more complex reality, in regard to either the stimuli that initiate aggregation or the modulating effects of fluid dynamic forces. For example, the notion that fibrinogen binding to $\alpha_{\mathrm{IIb}} \beta_{3}$ represents the only interaction relevant for aggregation, long a dogma in the field, relies on the study of stimulated platelets in stirred suspensions (12). This condition reflects only blood flowing at low velocity, such as in veins, and provides results that are not applicable to all areas of the circulation. Indeed, von Willebrand factor (vWF) can substitute for fibrinogen as the $\alpha_{\mathrm{IIb}} \beta_{3}$ ligand mediating platelet aggregation (13-16), and vWF becomes absolutely required when platelets aggregate under the effect of elevated shear stress in the absence of exogenous

\footnotetext{
Figure 1

Interactions proposed to mediate platelet adhesion and aggregation during thrombus formation. Events are depicted from left to right as they may occur in temporal sequence, initiating with platelet tethering to a reactive surface. At shear rates of less than $500-1,000 \mathrm{~s}^{-1}$, stable adhesion may occur independently of the initial vWF-GP Ib $\alpha$ interaction. The scheme considers only known adhesive interactions and does not exclude the relevance of other ligand-receptor pairs for platelet thrombus formation and other agonists in platelet activation. The 2 arrows connecting activation and stable adhesion express the hypothesis that activation usually precedes stable adhesion, particularly when thrombus formation occurs under the influence of high shear stress, but specific adhesive bonds may also enhance activation. (Modified from Savage et al. [10] and reprinted with permission.)
}

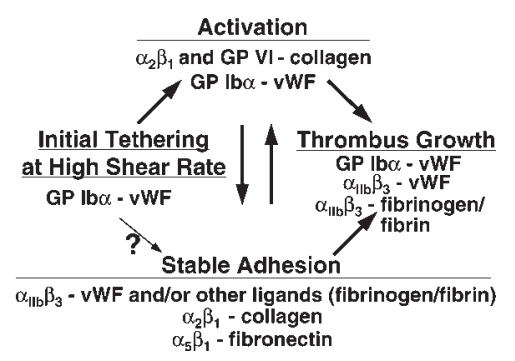


agonists $(17,18)$. There is also good evidence that whenever vWF mediates stable surface adhesion (19) or aggregation $(11,18)$ of platelets, it must engage both of its platelet receptors (GP Ib $\alpha$ in the GP Ib-IX-V complex and $\alpha_{\mathrm{IIb}} \beta_{3}$ ) sequentially (15). The synergism between these 2 receptors occurs irrespective of shear forces (19) and can even be demonstrated under static conditions (20). Nevertheless, only high shear stress conditions in rapidly flowing blood, as found in arterioles or larger arteries with obstructed lumina, highlight the indispensable role of vWF in adhesion and aggregation. At high shear rates, only the bond between the vWF A1 domain and GP Ib $\alpha$ can initiate the capture and tethering of platelets to a surface, whether an ECM component (10) or another platelet (11). However, this interaction is intrinsically short-lived. By itself, it can only mediate platelet rolling and does not result in firm adhesive contacts (19), which are typically supported by binding of integrins to $\mathrm{VWF}$ or other substrates $(10,11)$.

In this issue of the JCI, Kulkarni and co-workers discuss a revised model of platelet aggregation (21). They also confirm mechanisms that have been well outlined in other studies, demonstrating in particular that platelet-bound vWF contributes to platelet recruitment into a growing thrombus. The vWF present at a site of vascular injury originates both from plasma and from platelet $\alpha$-granules, the latter being released after activation $(22,23)$. Moreover, vWF acutely released from endothelial cells, under the influence of fibrin or other stimuli associated with a vascular lesion (24), may also act locally before diffusing into the circulating pool (25). In any case, plasma vWF appears to play a central role in thrombus formation under high shear stress conditions, because it can mediate initial steps in the process, even before the release of $\alpha$-granule or endothelial proteins. This may explain, for example, the apparent association between elevated circulating vWF and increased risk of acute coronary artery occlusion (26). Regardless of its origin, vWF may not act alone under conditions of high shear stress to link platelets to one another. Fibrinogen (or fibrin) also apparently helps stabilize the forming thrombus, even under conditions in which vWF is absolutely required to initiate platelet recruitment $(11,27)$. Thus, at both early and late stages of thrombus formation, platelet adhesive mechanisms in rapidly flowing blood depend on multiple synergistic bonds, involving different receptors and ligands with specific functions (Figure 2).

Kulkarni and co-workers also conclude that $\mathrm{vWF}$ participates in platelet function even at shear rates lower than those typical of the arterial circulation (21). This is possible because, as already discussed here, all the necessary interactions involving immobilized vWF and its platelet receptors can take place irrespective of hemodynamic conditions. In an experimental setting, such phenomena can be effectively demonstrated by creating an environment in which vWF is the predominant adhesive ligand, as in the case of a monolayer of activated platelets (21). In an intact organism, however, other adhesive proteins are available that can effectively support the recruitment of platelets onto a

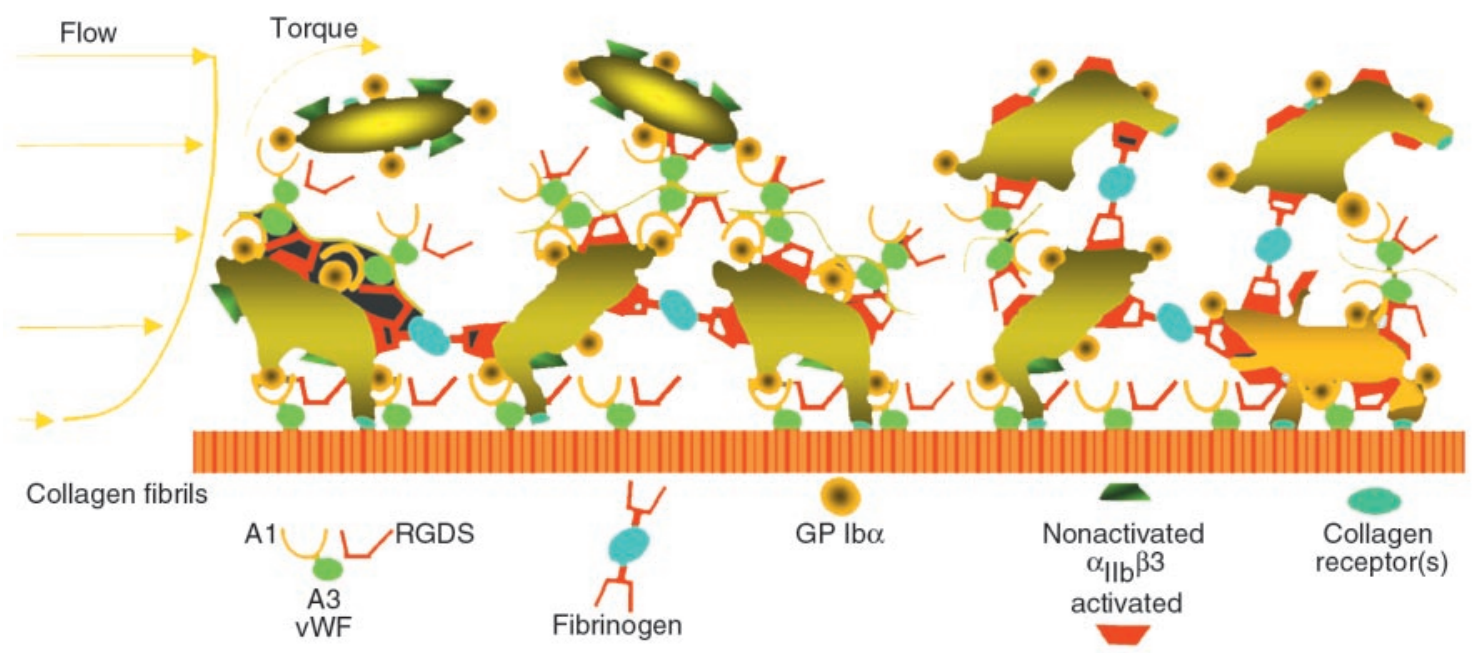

Figure 2

Schematic representation of the mechanisms of platelet adhesion and aggregation in flowing blood. In a cylindrical vessel, the velocity profile of particles contained in circulating blood is parabolic; the shear rate decreases from the wall to the center of the lumen inversely to the flow velocity. In a flow field with high shear rate, only GP Ib $\alpha$ interaction with immobilized vWF multimers can initiate the tethering of circulating platelets to the vessel wall and to already adherent platelets. This GP Ib $\alpha$-dependent interaction supports initially transient bonds, depicted by the ongoing detachment of the 2 top platelets from vWF multimers bound to already activated platelets. The process is amplified by the activation of $\alpha_{\| 1} \beta_{3}$, which may occur during the transient tethering or through the action of other receptors that bind collagen or other components of exposed vascular or extravascular surfaces (see also Figure 1). The final result is stable attachment of recruited platelets and irreversible membrane binding of soluble adhesive ligand (fibrinogen and VWF), thus providing the substrate for additional recruitment of nonactivated platelets and leading to thrombus growth. Note that nonactivated $\alpha_{1 \mathrm{lb}} \beta_{3}$ cannot bind soluble ligands. The bridging effect of fibrinogen, which is required to stabilize platelet aggregation and resist the effects of high shear stress, only occurs after initial tethering of platelets through the interaction of VWF and GP Ib $\alpha$. At shear rates less than 500-1,000 s-1, the adhesive functions of vWF are no longer indispensable, either for initial attachment to a thrombogenic surface or for aggregation. Thus, even in the absence of vWF, collagen receptors (among others) can permit stable adhesive interactions to form rapidly, and fibrin or fibrinogen can bind to platelets to permit aggregation. 
thrombogenic surface and mediate aggregation even without the participation of $\mathrm{vWF}$, provided that shear rates do not exceed certain limits.

In this regard, it is important to realize that the stated values for shear rates in experimental flow models may be easily misinterpreted. Reported shear rates typically indicate values at the surface exposed to laminar flow before platelet deposition occurs. A growing thrombus significantly alters these initial conditions, because shear rates increase where the flow path becomes restricted, and areas of disturbed flow may appear where the streaming fluid separates around an obstacle. Thus, vWF may not be required to initiate platelet adhesion and aggregation in experiments conducted at a nominal wall shear rate of $300 \mathrm{~s}^{-1}$, but its functional inhibition limits the height reached by thrombi (11), because hemodynamic forces are greater at the tip of a growing thrombus. As thrombus height increases levels of shear stress may exceed the limit at which fibrinogen-dependent binding is successful. At this point, further platelet recruitment is impaired unless initial tethering can occur through the binding of vWF and GP Ib $\alpha$ (11) (Figure 2).

In spite of considerable advances during the last several years, key aspects of the mechanisms that regulate platelet function in hemostasis and thrombosis remain to be elucidated. Experimental models in genetically altered animals indicate that other, still unidentified adhesive ligands may play a direct role in mediating some degree of platelet aggregation even in the absence of fibrinogen and $\mathrm{VWF}$ (28). The interpretation of such findings, yet to be reported in full detail, may not be straightforward. No condition is known in which both vWF and fibrinogen are deficient in humans, and it is possible that the absence of these 2 key platelet ligands permits interactions to occur that would otherwise be functionally irrelevant. Nevertheless, observations of this kind have the exciting potential to identify new components of ECM and/or blood capable of initiating or inhibiting the response of platelets at sites of vascular injury. Mouse models have provided unexpected findings, for example with respect to the involvement of thrombospondin-2 in hemostasis (29). The sudden occurrence of acute arterial occlusion, such as in the coronary arteries, is likely determined by the rate of thrombus growth and may be influenced by the nature and relative abundance in the vessel wall of different substrates for platelet adhesion and activation. The ECM composition may vary as a result of inflammatory processes developing in an atherosclerotic lesion (30), and plaque rupture may have different outcomes depending on which substrates become exposed to flowing blood.

Future research should offer a more global view of the processes underlying hemostasis and thrombosis. To this end, the adhesive mechanisms that support platelet function and the signals that activate platelets or dampen their responses (31) must be considered in association with the reactions that lead to fibrin deposition and activate anticoagulant and fibrinolytic pathways on platelet and vascular surfaces. With appropriate experimental models, the results of such studies will enhance our ability to understand, diagnose, and treat disturbances of platelet function.

1. Italiano, J.E., Jr., Lecine, P., Shivdasani, R.A., and Hartwig, J.H. 1999. Blood platelets are assembled principally at the ends of the proplatelet process es produced by differentiated megakaryocytes. $J$. Cell Biol. 147:1299-1232.

2. Weiss, H.J. 1975. Platelet physiology and abnormalities of platelet function (first of two parts). N. Engl. J. Med. 293:531-541.

3. Weiss, H.J. 1975. Platelet physiology and abnormalities of platelet function (second of two parts). N. Engl. J. Med. 293:580-588.

4. Sixma, J.J., and Wester, J. 1977. The hemostatic plug. Semin. Hematol. 14:265-299.

5. Fuster, V., Badimon, L., Badimon, J.J., and Chesebro, J.H. 1992. The pathogenesis of coronary artery disease and the acute coronary syndromes (1). N. Engl. J. Med. 326:242-250.

6. Fuster, V., Badimon, L., Badimon, J.J., and Chesebro, J.H. 1992. The pathogenesis of coronary artery disease and the acute coronary syndromes (2). N. Engl. J. Med. 326:310-318.

7. Coller, B.S. 1995. Blockade of platelet GPIIb/IIIa receptors as an antithrombotic strategy. Circulation. 92:2373-2380.

8. Coller, B.S. 1997. Platelet GPIIB/IIIa antagonists: the first anti-integrin receptor therapeutics. $J$. Clin. Invest. 99:1467-1471.

9. Madan, M., Berkowitz, S.D., and Tcheng, J.E. 1998. Glycoprotein IIb/IIIa integrin blockade. Circulation. 98:2629-2635.

10. Savage, B., Almus-Jacobs, F., and Ruggeri, Z.M. 1998. Specific synergy of multiple substratereceptor interactions in platelet thrombus formation under flow. Cell. 94:657-666.

11. Ruggeri, Z.M., Dent, J.A., and Saldivar, E. 1999. Contribution of distinct adhesive interactions to platelet aggregation in flowing blood. Blood. 94:172-178.

12. Born, G.V.R. 1962. Aggregation of blood platelets by adenosine diphosphate and its reversal. Nature. 194:927-929.

13. Ruggeri, Z.M., Bader, R., and De Marco, L. 1982 Glanzmann thrombasthenia: deficient binding of von Willebrand factor to thrombin-stimulated platelets. Proc. Natl. Acad. Sci. USA. 79:6038-6041.

14. Fujimoto, T., and Hawiger, J. 1982. Adenosine diphosphate induces binding of von Willebrand factor to human platelets. Nature. 297:154-156.

15. Ruggeri, Z.M., De Marco, L., Gatti, L., Bader, R. and Montgomery, R.R. 1983. Platelets have more than one binding site for von Willebrand factor. J. Clin. Invest. 72:1-12.

16. De Marco, L., Girolami, A., Zimmerman, T.S., and Ruggeri, Z.M. 1986. von Willebrand factor interaction with the glycoprotein IIb/IIIa complex. Its role in platelet function as demonstrated in patients with congenital afibrinogenemia. J. Clin. Invest. 77:1272-1277.

17. Peterson, D.M., Stathopoulos, N.A., Giorgio, T.D., Hellums, J.D., and Moake, J.L. 1987. Shearinduced platelet aggregation requires von Willebrand factor and platelet membrane glycoproteins Ib and IIb-IIIa. Blood. 69:625-628.

18. Goto, S., Ikeda, Y., Saldivar, E., and Ruggeri, Z.M 1998. Distinct mechanisms of platelet aggregation as a consequence of different shearing flow conditions. J. Clin. Invest. 101:479-486.

19. Savage, B., Saldivar, E., and Ruggeri, Z.M. 1996 Initiation of platelet adhesion by arrest onto fibrinogen or translocation on von Willebrand factor. Cell. 84:289-297.

20. Savage, B., Shattil, S.J., and Ruggeri, Z.M. 1992 Modulation of platelet function through adhesion receptors: a dual role for glycoprotein IIbIIIa (integrin $\alpha_{\text {IIb }} \beta_{3}$ ) mediated by fibrinogen and glycoprotein Ib-von Willebrand factor. J. Biol. Chem. 267:11300-11306.

21. Kulkarni, et al. 2000. A revised model of platelet aggregation. J. Clin. Invest. 105:783-791.

22. Mannucci, P.M., et al. 1987. Correction of the bleeding time in treated patients with severe von Willebrand disease is not solely dependent on the normal multimeric structure of plasma von Willebrand factor. Am. J. Hematol. 25:55-65.

23. Castillo, R., et al. 1991. Hemostatic effect of normal platelet transfusion in severe von Willebrand disease patients. Blood. 77:1901-1905.

24. Ribes, J.A., Francis, C.W., and Wagner, D.D. 1987. Fibrin induces release of von Willebrand factor from endothelial cells. J. Clin. Invest. 79:117-123.

25. Sporn, L.A., Marder, V.J., and Wagner, D.D. 1986 Inducible secretion of large, biologically potent von Willebrand factor multimers. Cell. 46:185-190.

26. Goto, S., et al. 1999. Enhanced shear-induced platelet aggregation in acute myocardial infarction. Circulation. 99:608-613.

27. Tsuji, S., et al. 1999. Real-time analysis of mural thrombus formation in various platelet aggregation disorders: distinct shear-dependent roles of platelet receptors and adhesive proteins under flow. Blood. 94:968-975.

28. Ni, H., et al. 1999. Distinct roles of von Willebrand factor and fibrinogen in thrombus growth in arterioles. Thromb. Haemost. Suppl. p. 411. (Abstr.)

29. Kyriakides, T.R., et al. 1998. Mice that lack thrombospondin 2 display connective tissue abnormalities that are associated with disordered collagen fibrillogenesis, an increased vascular density, and a bleeding diathesis. J. Cell Biol. 140:419-430.

30. Ross, R. 1999. Atherosclerosis: an inflammatory disease. N. Engl. J. Med. 340:115-126.

31. Marcus, A.J., et al. 1997. The endothelial cell EctoADPase responsible for inhibition of platelet function is CD39. J. Clin. Invest. 99:1351-1360. 